\title{
Chimeras and Clusters Emerging from Robust-Chaos Dynamics
}

\author{
M. G. Cosenza $\mathbb{D}^{1,2}$ O. Alvarez-Llamoza, ${ }^{3}$ and A. V. Cano ${ }^{4,5}$ \\ ${ }^{1}$ School of Physical Sciences \& Nanotechnology, Universidad Yachay Tech, Urcuquí, Ecuador \\ ${ }^{2}$ Universidad de Los Andes, Mérida, Venezuela \\ ${ }^{3}$ Grupo de Simulación, Modelado, Análisis y Accesabilidad, Universidad Católica de Cuenca, Cuenca, Ecuador \\ ${ }^{4}$ Institute for Integrative Biology, ETH, Zurich, Switzerland \\ ${ }^{5}$ Swiss Institute of Bioinformatics, Lausanne, Switzerland \\ Correspondence should be addressed to M. G. Cosenza; mario.cosenza@gmail.com
}

Received 10 August 2020; Revised 19 January 2021; Accepted 3 February 2021; Published 15 February 2021

Academic Editor: Lucia Valentina Gambuzza

Copyright $\odot 2021$ M. G. Cosenza et al. This is an open access article distributed under the Creative Commons Attribution License, which permits unrestricted use, distribution, and reproduction in any medium, provided the original work is properly cited.

We show that dynamical clustering, where a system segregates into distinguishable subsets of synchronized elements, and chimera states, where differentiated subsets of synchronized and desynchronized elements coexist, can emerge in networks of globally coupled robust-chaos oscillators. We describe the collective behavior of a model of globally coupled robust-chaos maps in terms of statistical quantities and characterize clusters, chimera states, synchronization, and incoherence on the space of parameters of the system. We employ the analogy between the local dynamics of a system of globally coupled maps with the response dynamics of a single driven map. We interpret the occurrence of clusters and chimeras in a globally coupled system of robust-chaos maps in terms of windows of periodicity and multistability induced by a drive on the local robust-chaos map. Our results show that robustchaos dynamics does not limit the formation of cluster and chimera states in networks of coupled systems, as it had been previously conjectured.

\section{Introduction}

Many smooth nonlinear dynamical systems possess chaotic attractors embedded with a dense set of periodic orbits for any range of parameter values. Therefore, in practical systems operating in chaotic mode, a slight perturbation of a parameter may drive the system out of chaos. Alternatively, there exist dynamical systems that exhibit the property of robust chaos [1-6]. A chaotic attractor is said to be robust if, for its parameter values, there exists a neighborhood in the parameter space where windows of periodic orbits are absent and the chaotic attractor is unique [1].

Robust chaos constitutes an advantageous feature in applications that require reliable functioning in a chaotic regime, in the sense that the chaotic behavior cannot be removed by arbitrarily small fluctuations of the system parameters. For instance, networks of coupled maps with robust chaos have been efficiently used in communication and encryption algorithms [7] and they have been investigated for information transfer across scales in complex systems [8]. In addition, the existence of robust chaos allows for heterogeneity in the local parameters of a system of coupled oscillators, while guaranteeing the performance of all the oscillators in a chaotic mode.

On the other hand, systems possessing robust chaos may present limitations in the types of collective behaviors that they can achieve, in comparison with systems displaying periodic windows. For example, it has been conjectured that the phenomenon of dynamical clustering in globally coupled networks (where the system segregates into distinguishable subsets of synchronized elements) is only found when stable periodic windows are present in the local elements [9-11]. Recently, it has also been argued that chimera states (i.e., coexistence of subsets of oscillators with synchronous and asynchronous dynamics) cannot emerge in networks of coupled oscillators having robust chaotic attractors [12, 13].

The phenomenon of dynamical clustering is relevant as it can provide a simple mechanism for the emergence of differentiation, segregation, and ordering of elements in many physical and biological systems $[14,15]$. Clustering has 
been found in systems of globally coupled Rössler oscillators [16], neural networks [17], and biochemical reactions [18] and has been observed experimentally in arrays of globally coupled electrochemical oscillators [19] and globally coupled salt-water oscillators [20]. In addition, the study of chimera states currently attracts much interest (for reviews see $[21,22]$ ). Chimera states have been found in networks of nonlocally coupled phase oscillators [23,24], in systems with local [25-28] and global [29-34] interactions, and in networks of time-discrete maps [35-38]. These states have been investigated in a diversity of contexts [39-47]. Chimera states have been observed in experimental settings, such as populations of chemical oscillators [48], coupled lasers [49], optical light modulators [50], and electronic [51] and mechanical $[52,53]$ oscillator systems. It has been shown that clustering is closely related to the formation of chimera states in systems of globally coupled periodic oscillators [31].

In this paper, we investigate the occurrence of dynamical clustering and chimera states in systems of coupled robustchaos oscillators. In Section 2, we describe the characterization of synchronization and cluster and chimera states in globally coupled systems. In Section 3, we consider a network of globally coupled robust-chaos maps and show that cluster and chimera states can actually emerge in this system for several values of parameters. In Section 4, we employ the analogy between the local dynamics of the globally coupled system with the response dynamics of a single driven map. We interpret the occurrence of clusters and chimeras in the globally coupled system in terms of windows of periodicity induced by the drive on the local robust-chaos map. Conclusions are presented in Section 4.

\section{Methods}

A global interaction in a system can be described as a field or influence acting on all the elements in the system. As a simple model of an autonomous dynamical system subject to a global interaction, we consider a system of $N$ maps coupled in the form

$$
x_{t+1}^{i}=(1-\varepsilon) f\left(x_{t}^{i}\right)+\varepsilon h_{t}\left(x_{t}^{j} \mid j \in S\right),
$$

where $x_{t}^{i}(i=1,2, \ldots, N)$ describes the state variable of the $i$ th map in the system at discrete time $t$, the function $f$ expresses the local dynamics of the maps, the function $h_{t}$ represents a global field that depends on the states of the elements in a given subset $S$ of the system, at time $t$, and the parameter $\varepsilon$ measures the strength of the coupling of the maps to the field. The form of the coupling in equation (1) is assumed in the commonly used diffusive form. The function $h_{t}$ may not depend on all the elements, but it must be shared by all the elements of the system to be a global interaction.

A collective state of synchronization or coherence takes place in system equation (1) when $x_{t}^{i}=x_{t}^{j}, \forall i, j$ for asymptotic times. A desynchronized or incoherent state corresponds to $x_{t}^{i} \neq x_{t}^{j}, \forall i, j$ for all times. Dynamical clustering occurs when the system segregates into a number of $K$ distinct clusters or subsets of elements such that elements in given subset are synchronized among themselves. In other words, $x_{t}^{i}=x_{t}^{j}=$
$X_{t}^{\xi}, \forall i, j$ in the $\xi$ th cluster, where $X_{t}^{\xi}$ denotes the value of $x_{t}^{i}$ in that cluster, with $\xi=1, \ldots, K$. If $n_{\xi}$ is the number of elements belonging to the $\xi$ th cluster, then its relative size is $p_{\xi}=n_{\xi} / N$. In general, the number of clusters, their size, and their dynamical evolution (periodic, quasiperiodic, or chaotic) depend on the initial conditions and parameters of the system. A chimera state consists of the coexistence of one or more clusters and a subset of desynchronized elements. If there are $K$ clusters, the fraction of elements in the system belonging to clusters is $p=\sum_{\xi=1}^{K} n_{\xi} / N$ while $(1-p)$ is the fraction of elements in the desynchronized subset.

In practical applications, we consider that two elements $i$ and $j$ belong to a cluster at time $t$ if the distance between their state variables, defined as

$$
d_{i j}(t)=\left|x_{t}^{i}-x_{t}^{j}\right|,
$$

is less than a threshold value $\delta$, i.e., if $d_{i j}<\delta$. The choice of $\delta$ should be appropriate for achieving differentiation between closely evolving clusters. Then, we calculate the fraction of elements that belong to some cluster at time $t$ as [16]

$$
p(t)=1-\frac{1}{N} \sum_{i=1}^{N} \prod_{j=1, j \neq i}^{N} \Theta\left[d_{i j}(t)-\delta\right],
$$

where $\Theta(x)=0$ for $x<0$ and $\Theta(x)=1$ for $x \geq 0$. We refer to $p$ as the asymptotic time average of $p(t)$. Then, a clustered state in the system can be characterized by the value $p=1$, while an incoherent state in the system corresponds to $p \longrightarrow 0$. The values $p_{\min }<p<1$ characterize a chimera state, where $p_{\min }$ is the minimum cluster size to be taken into consideration.

A synchronization state corresponds to the presence of a single cluster of size $N$ and has also the value $p=1$. To distinguish a synchronization state from a multicluster state, we calculate the asymptotic time average $\langle\sigma\rangle$ as

$$
\langle\sigma\rangle=\frac{1}{T-\tau} \sum_{t=\tau}^{T} \sigma_{t},
$$

where $\tau$ is the number of discarded transients, $T$ is a sufficiently large time, and $\sigma_{t}$ is the instantaneous standard deviation of the distribution of state variables defined by

$$
\sigma_{t}=\left[\frac{1}{N} \sum_{i=1}^{N}\left(x_{t}^{i}-\bar{x}_{t}\right)^{2}\right]^{1 / 2},
$$

where

$$
\bar{x}_{t}=\frac{1}{N} \sum_{j=1}^{N} x_{t}^{j} .
$$

Statistically, a synchronization state is characterized by the values $\langle\sigma\rangle=0$ and $p=1$, while a cluster state corresponds to $\langle\sigma\rangle>0$ and $p=1$. Chimera states are characterized by $\langle\sigma\rangle>0$ and $p_{\min }<p<1$, and desynchronization is described by $\langle\sigma\rangle>0, p<p_{\min }$. In this paper, we set $\delta=10^{-6}$ and $p_{\min }=0.05$.

Note that, in systems with local or long-range interactions where there is a natural spatial ordering, the 
synchronized and desynchronized domains for chimera states are localized in space. In contrast, globally coupled systems lack the notion of spatial order. Thus, the chimera and cluster states in our system are characterized in terms of the statistical quantities $\langle\sigma\rangle$ and $p$, not by the spatial location of the synchronized and the desynchronized domains.

\section{Results and Discussion}

3.1. Chimeras and Clusters in Globally Coupled Robust-Chaos Maps. Let us consider a network of globally coupled maps described by the equations [14]

$$
x_{t+1}^{i}=(1-\varepsilon) f\left(x_{t}^{i}\right)+\frac{\varepsilon}{N} \sum_{j=1}^{N} f\left(x_{t}^{j}\right),
$$

where the global interaction function is the mean field of the system, and

$$
h_{t}=\frac{1}{N} \sum_{j=1}^{N} f\left(x_{t}^{j}\right) .
$$

As local dynamics exhibiting robust chaos, we consider the following smooth, unimodal map defined on the interval $x \in[0,1][54]$ :

$$
x_{t+1}=f\left(x_{t}\right)=\frac{1-b^{\left(1-x_{t}\right) x_{t}}}{1-b^{1 / 4}},
$$

which is chaotic with no periodic windows on the parameter interval $b \in[0,1]$. On this interval, the Lyapunov exponent of map equation (9) has the constant value $\lambda=\ln 2$. The bifurcation diagram of map equation (9) in Figure 1 shows the absence of periodicity in the interval $b \in[0,1]$.

Figure 2 shows the asymptotic temporal evolution of the states of system equations (7) and (9), for different values of parameters. Since the system is globally coupled, there is no natural spatial ordering. For visualization purposes, the indexes $i$ are ordered at time $t=10^{4}$ such that $i<j$ if $x_{t}^{i}<x_{t}^{j}$ and kept fixed afterwards. The values of the states $x_{t}^{i}$ are represented by distinct color coding; two elements $i, j$ share the same color if $x_{t}^{i}=x_{t}^{j}$. A desynchronized state is displayed in Figure 2(a) and a complete synchronization state occurs in Figure 2(d), while a chimera state and a two-cluster state are visualized in Figures 2(b) and 2(c), respectively.

Figure 3 shows the collective states arising in system equations (7) and (9) on the space of parameters $\varepsilon, b$, characterized through the quantities $p$ and $\langle\sigma\rangle$. Labels indicate the regions where these behaviors occur: CS: complete synchronization; C: cluster states; Q: chimera states, and D: desynchronization.

The linear stability analysis for the complete synchronization state in globally coupled system equation (7) shows that this state is stable if the following condition is satisfied [14]:

$$
\left|(1-\varepsilon) e^{\lambda}\right|<1
$$

where $\lambda$ is the Lyapunov exponent for the local map $f(x)$. For map equation (9), we obtain that the completely synchronized state is stable for $1 / 2<\varepsilon<3 / 2$, which agrees with the numerical characterization for this state performed in Figure 3 . Figure 3 reveals that both cluster and chimera states can arise in globally coupled map networks for appropriate values of parameters, even when the individual maps lack periodic windows. Clusters and chimera state regions occur adjacent to each other for an intermediate range of values of the coupling parameter $\varepsilon$ on the phase diagram of Figure 3. In fact, chimeras and clusters are closely related collective states in systems subject to global interactions [30]. Chimera states appear to mediate between dynamical clustering and incoherence.

Multicluster chimera states are also possible in systems of globally coupled robust-chaos maps. As an illustration, consider the smooth unimodal map [55]

$$
f\left(x_{t}\right)=\sin ^{2}\left(r \arcsin \left(\sqrt{x_{t}}\right)\right),
$$

defined on the interval $x_{t} \in[0,1]$ for parameter values $r>1$. Figure 4 shows the bifurcation diagram of the iterates of map equation (11) as a function of the parameter $r$. The dynamics of the map displays robust chaos with no periodic windows for $r>1$. The Lyapunov exponent is $\lambda=\ln r$ [55].

Figure 5 shows the temporal evolution of the states of globally coupled system equation (7) with local map equation (11), for different values of parameters. A chimera state with multiple clusters occurs in Figure 5(a), while a two-cluster state is shown in Figure 5(b). Multichimera states or multiheaded chimeras (coexistence of multiple localized domains of incoherence and coherence) have been reported in systems with long-range interactions [56]. However, those states are not equivalent to a chimera state with multiple clusters in a globally coupled system, such as Figure 5(a), where there is no notion of locality.

3.2. Dynamics of Clusters and Chimera States with Global Interactions. Consider a chimera state consisting of $K$ clusters and a desynchronized subset in the system of globally coupled map equation (1). The dynamics of this state can be described by the equations

$$
\begin{aligned}
X_{t+1}^{\xi} & =(1-\varepsilon) f\left(X_{t}^{\xi}\right)+\varepsilon h_{t}, \quad \xi=1, \ldots, K, \\
x_{t+1}^{j} & =(1-\varepsilon) f\left(x_{t}^{j}\right)+\varepsilon h_{t}, \quad j=1, \ldots,(1-p) N .
\end{aligned}
$$

The mean field equation (8) in a chimera state can be expressed as the sum of two contributions

$$
h_{t}=h_{C}+h_{I}
$$

where

$$
\begin{aligned}
& h_{C}=\sum_{\xi=1}^{K} p_{\xi} f\left(X_{t}^{\xi}\right), \\
& h_{I}=\frac{1}{N} \sum_{j=1}^{(1-p) N} f\left(x_{t}^{j}\right) .
\end{aligned}
$$

The term $h_{C}$ is the contribution to the mean field corresponding to elements belonging to clusters, whereas $h_{I}$ is 


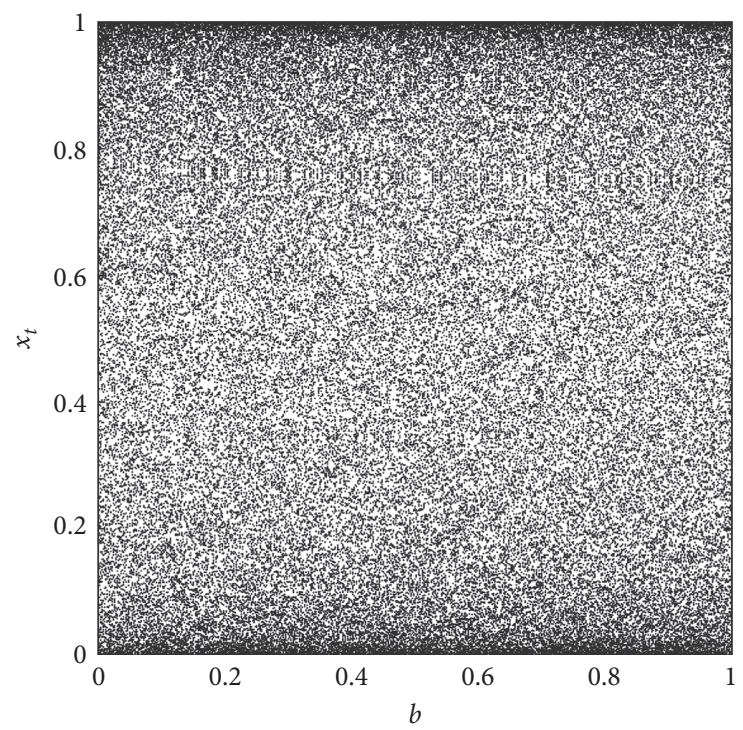

FIgURE 1: Bifurcation diagram of map equation (9) as a function of the parameter $b$.

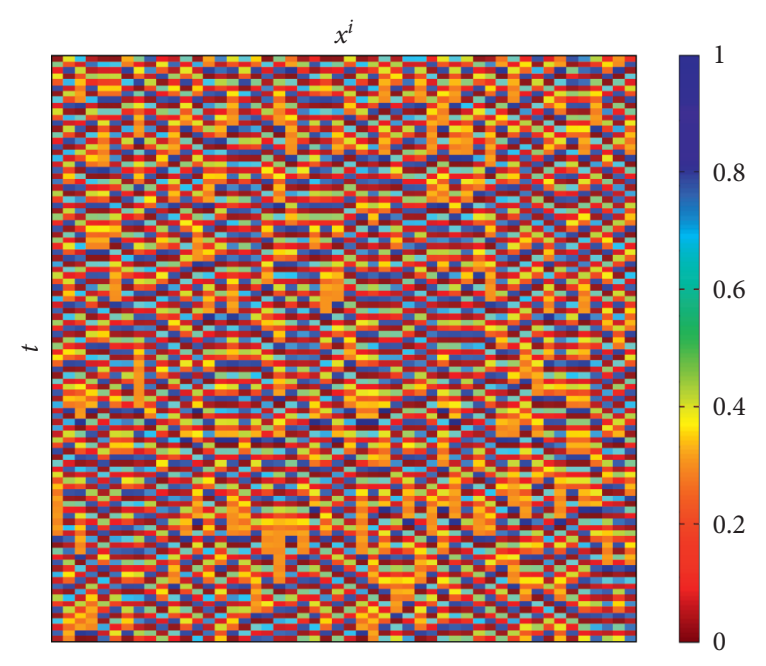

(a)

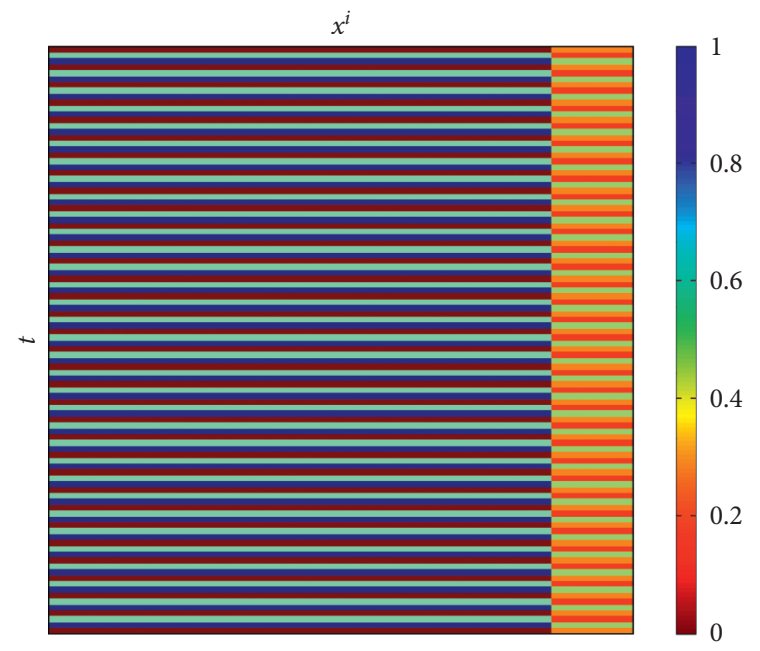

(c)

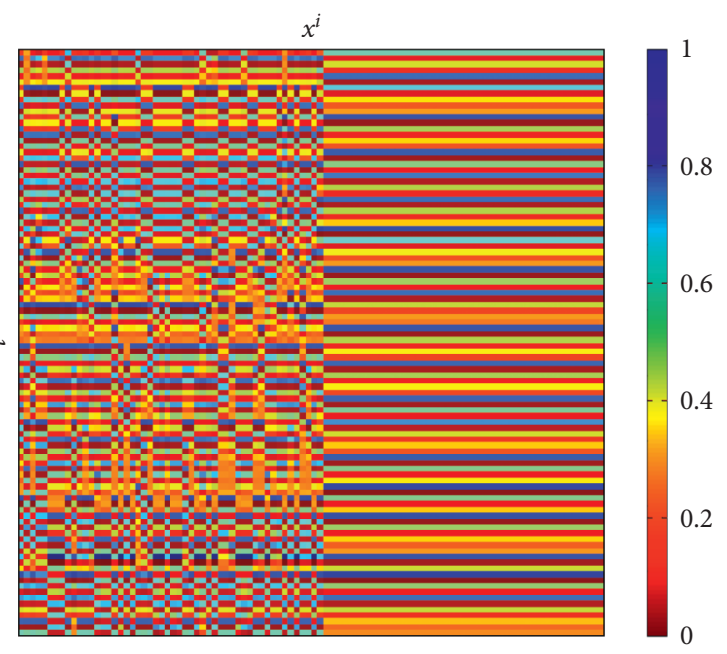

(b)

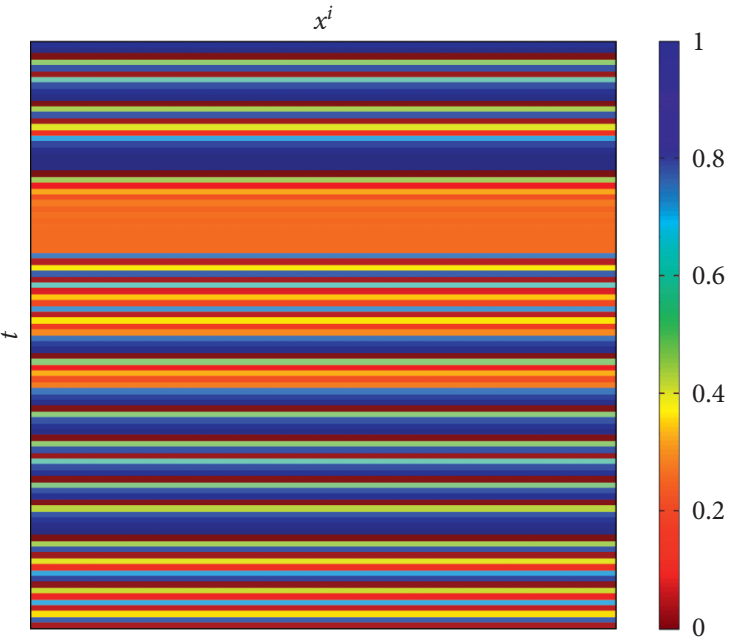

(d)

Figure 2: Asymptotic evolution of the states $x^{i}$ (horizontal axis) as a function of time (vertical axis) for system equations (7) and (9) with size $N=100$ and fixed $b=0.5$, for different values of the coupling parameter. Random initial conditions are uniformly distributed in the interval $[0,1]$. After discarding $10^{4}$ transients, 100 iterates $t$ are displayed. Ordering of the map indexes is explained in the text. Color code: two elements $i, j$ share the same color if $x_{t}^{i}=x_{t}^{j}$. (a) Incoherent or desynchronized state, $\varepsilon=0.15$. (b) Chimera state, $\varepsilon=0.2$. (c) Two-cluster state, $\varepsilon=0.39$. (d) Synchronization, $\varepsilon=0.6$. 


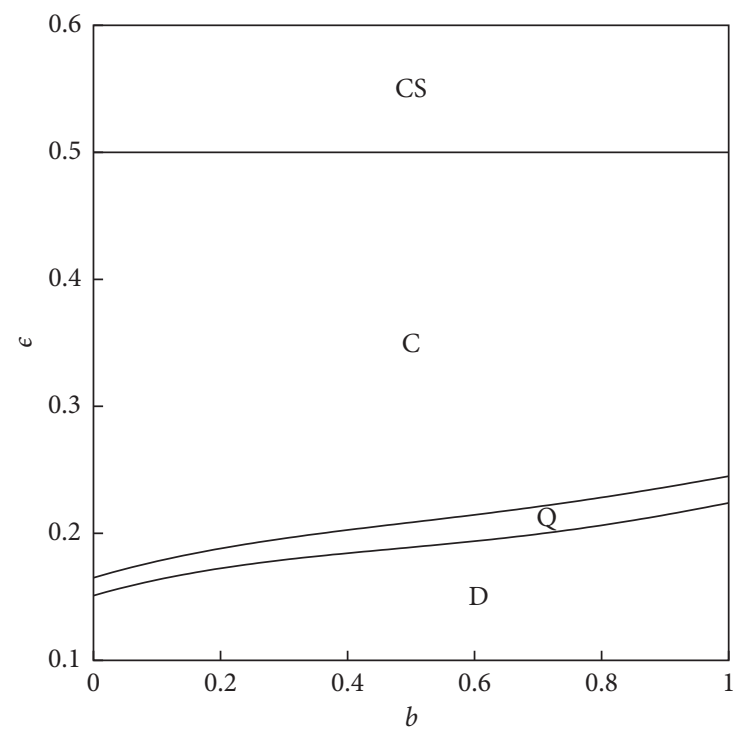

Figure 3: Phase diagram on the plane $(\varepsilon, b)$ for autonomous system equations (7) and (9) with size $N=500$. For each data point, the quantities $p$ and $\langle\sigma\rangle$ are obtained by averaging over 50 realizations of random initial conditions $x_{0}^{i}$ uniformly distributed in the interval $[0,1]$. Labels indicate different collective states. CS: synchronization; C: cluster states; Q: chimera states; D: desynchronization.

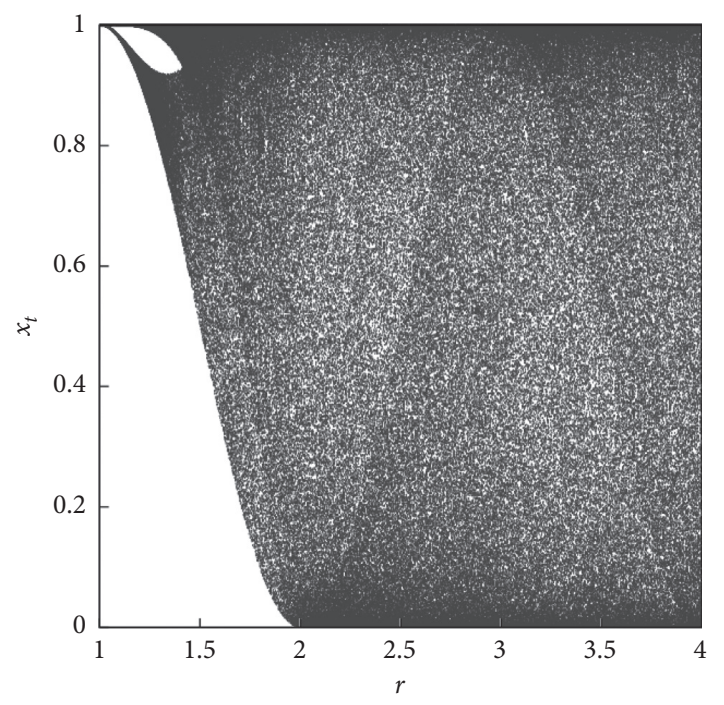

FiguRE 4: Bifurcation diagram of map equation (11) as a function of the parameter $r$.

the average of the states of the elements belonging to the incoherent subset.

Figure 6 shows the temporal behavior of both contributions $h_{C}$ and $h_{I}$ in a chimera state for globally coupled autonomous system equations (7) and (9). The time evolution of the cluster contribution $h_{C}$ is chaotic, similar to that of local map equation (9), but $h_{C}$ has a smaller amplitude. In general, the form of $h_{C}$ can be approximated as $h_{C} \approx A f\left(y_{t}\right)$, where $A<1$ represents a modulation factor reflecting the partition into several clusters. On the other hand, Figure 6 reveals that the time series of $h_{I}$ fluctuates about a mean value with a small dispersion, corresponding to the superposition of the dynamics of many incoherent chaotic elements. Thus, for the given parameter values, the incoherent contribution to the mean field for a large system size can be expressed approximately as a constant, i.e., $h_{I} \approx k$.

The dynamics of globally coupled system equation (1), where each map is subject to a feedback field $h_{t}$, can be compared to that of a replica system of maps subject to a global external drive $g\left(y_{t}\right)$ in the form

$$
\begin{aligned}
& x_{t+1}^{i}=(1-\varepsilon) f\left(x_{t}^{i}\right)+\varepsilon g\left(y_{t}\right), \\
& y_{t+1}=g\left(y_{t}\right) .
\end{aligned}
$$

It has been shown that an analogy between autonomous system equation (1) and driven system equation (15) can be established when the time evolution of the field $h_{t}$ is identical to that of the function $g\left(y_{t}\right)$ [9]. Then, the drive-response dynamics at the local level in both systems is similar, and 


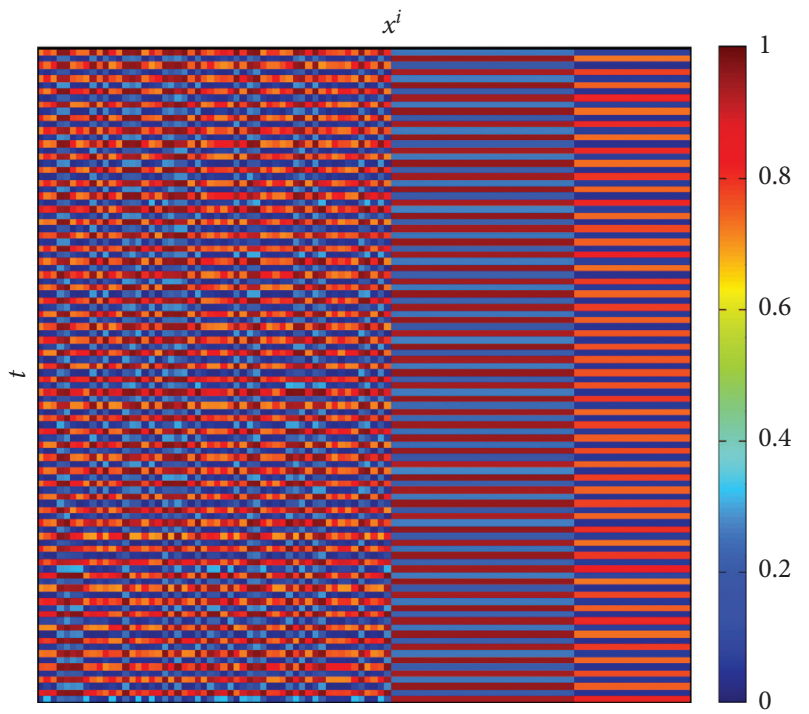

(a)

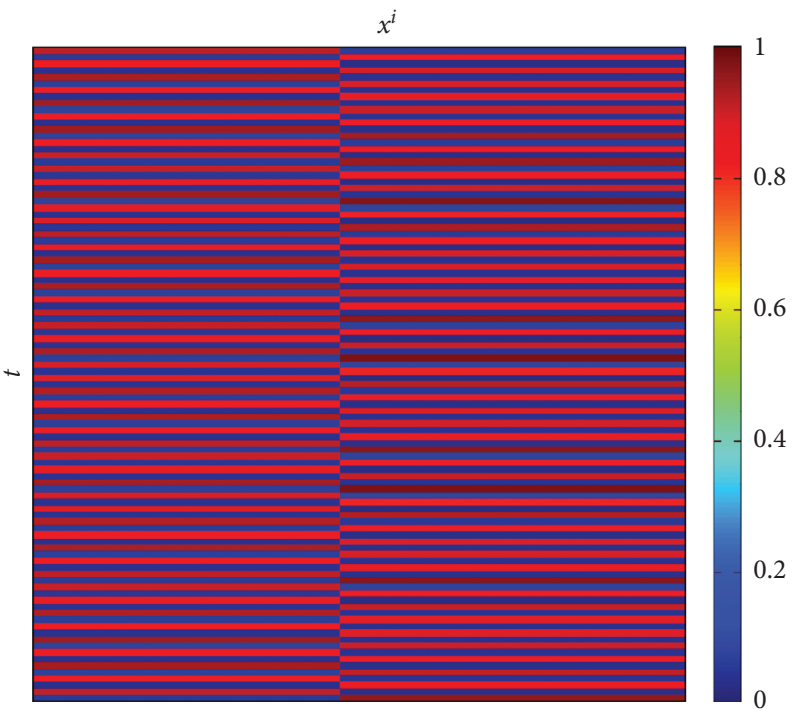

(b)

Figure 5: Asymptotic states $x^{i}$ (horizontal axis) as a function of time (vertical axis) for system equation (7) with size $N=100$ and local map equation (11), for different values of parameters. Initial conditions and ordering of the maps are similar to those in Figure 2. Color code: two elements $i, j$ share the same color if $x_{t}^{i}=x_{t}^{j}$. (a) Chimera state with two clusters, $r=3, \varepsilon=0.235$. (b) Two-cluster state, $r=3, \varepsilon=0.272$.

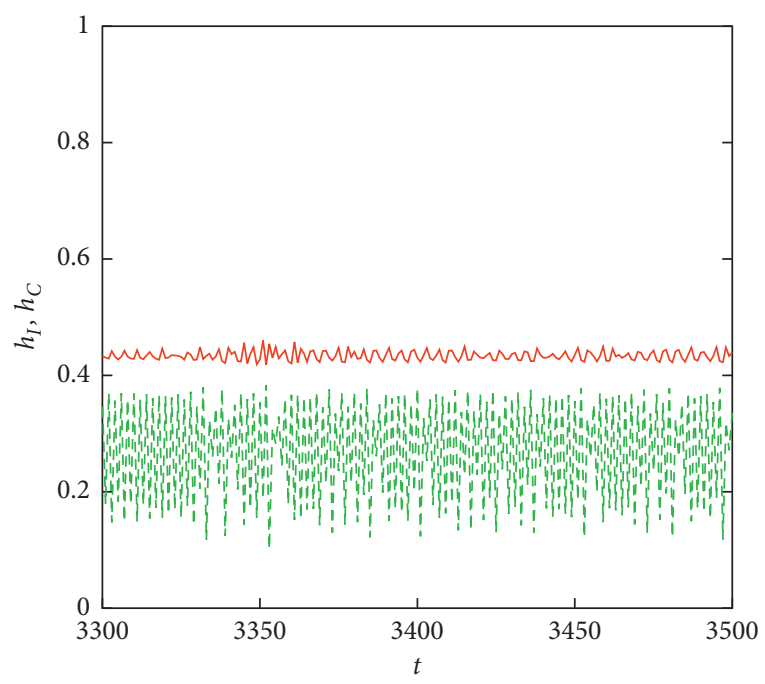

Figure 6: Cluster $h_{C}$ (green line) and incoherent $h_{I}$ (red line) contributions to the mean field of system equations (7) and (9), as functions of time. Fixed parameters $b=0.5$ and $\varepsilon=0.19$, and size $N=10^{4}$.

therefore their corresponding emerging collective states can be equivalent for some appropriate parameter values and initial conditions. In particular, chimera or cluster states in system equation (15) should be induced by an external drive function of the form $g\left(y_{t}\right)=A f\left(y_{t}\right)+k$, with $A, k$ constants, that imitates the mean field $h_{t}$. The realization of these states depends on the parameters $A$ and $k$ of the drive and on the coupling strength $\varepsilon$.

Figure 7 shows the temporal evolution of the states of driven system equation (15) with local map equation (9), for some values of parameters. A chimera state with a single cluster takes place in Figure 7 (a) for parameter values $(\varepsilon, b)$, where chimera states also occur in the autonomous system equations (7) and (9), as seen in the corresponding phase diagram of Figure 3. Figure 7(b) shows a two-cluster state for values $(\varepsilon, b)$ located in the region corresponding to clustered states in Figure 3 . The dynamics of the driven system equations (15) shows multistability; depending on initial conditions, chimeras with different partitions may be induced for given parameters values $(\varepsilon, b)$ in the region labeled $\mathrm{Q}$ in Figure 3. Similarly, different initial conditions may produce cluster states with different partition sizes for fixed parameter values in region $\mathrm{C}$ of Figure 3.

System equation (15) can be considered as $N$ realizations for different initial conditions of a single driven map

$$
\begin{aligned}
& x_{t+1}=(1-\varepsilon) f\left(x_{t}\right)+\varepsilon g\left(y_{t}\right), \\
& y_{t+1}=g\left(y_{t}\right) .
\end{aligned}
$$

Analogously, each local map in globally coupled system equation (7) can be seen as subject to a field $h_{t}$ that eventually induces a collective state. Clustering in globally coupled systems of identical elements has been attributed to the existence of periodic windows in the local dynamics [10]. On the other hand, clustering is considered a prerequisite for the occurrence of chimera states in globally coupled systems [31]. Thus, to elucidate the origin of clusters and chimeras in system equation (7) with local robust chaos, one can explore the response dynamics of the single driven map equation (16) with a function of the form $g\left(y_{t}\right)=A f\left(y_{t}\right)+k$ and $f$ having robust chaos. Then, if periodic windows are induced by the drive on a single map, one may expect that clusters and chimeras should arise in a globally coupled system of those maps. 


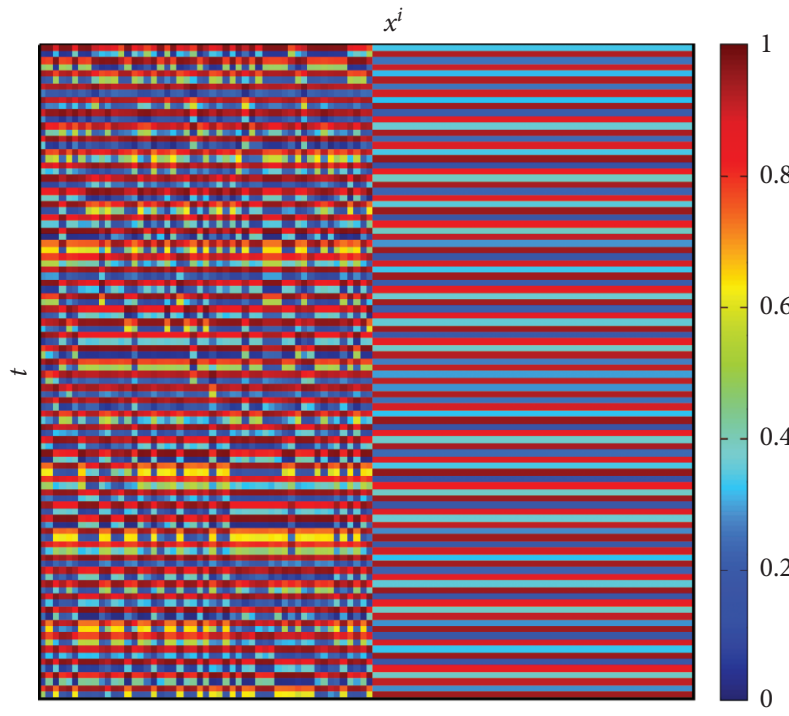

(a)

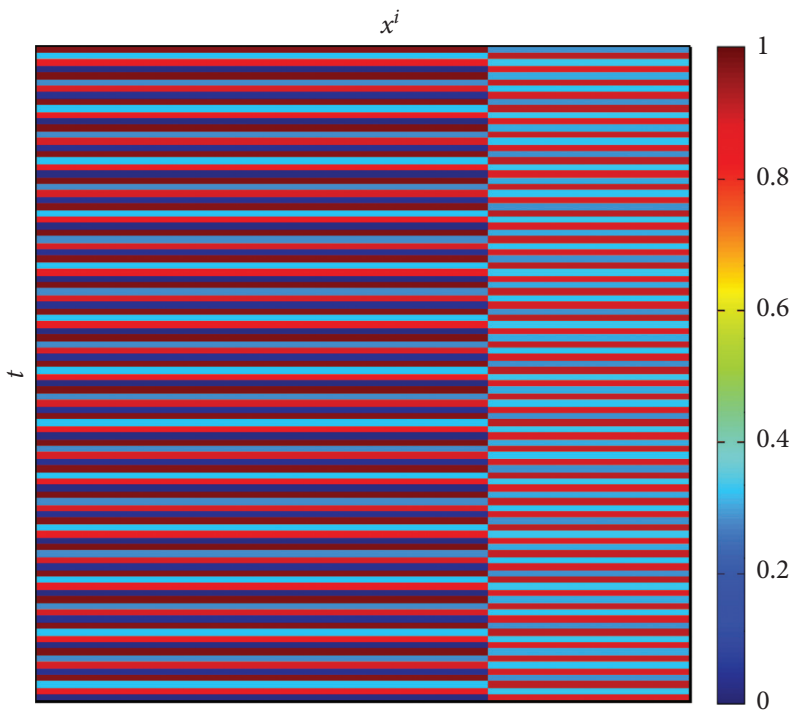

(b)

Figure 7: Asymptotic evolution of the states $x^{i}$ (horizontal axis) as a function of time (vertical axis) for driven system equation (15) with size $N=100$ and local map equation (9), for different values of the coupling parameter. Fixed values: $A=0.48, k=0.4, b=0.5$. Random initial conditions $x_{0}^{i}$ are uniformly distributed in the interval [0,1]. After discarding $10^{4}$ transients, 100 iterates $t$ are displayed. Ordering of the maps is similar to that in Figure 2. Color code: two elements $i, j$ share the same color if $x_{t}^{i}=x_{t}^{j}$. (a) Chimera state, $\varepsilon=0.198$. (b) Two-cluster state, $\varepsilon=0.272$.

Even a trivial function $g$ can modify the dynamics of a driven robust-chaos map in equation (16) to produce periodic windows. Figure $8(a)$ shows the bifurcation diagram of $x_{t}$ in equation (16) versus $\varepsilon$ for the map $f$ given by equation (9) with $g\left(y_{t}\right) \longrightarrow 0$, which is equivalent to a rescaling of $f$. Periodic windows typical of unimodal maps appear in the rescaled map $x_{t+1}=(1-\varepsilon) f\left(x_{t}\right)$. In general, the driven map equation (16) represents a rescaling of the robust-chaos map $f$ that acquires periodic windows. Similarly, the periodic cluster states arising in the globally coupled system equations (7) and (9) are a consequence of the windows of periodicity induced locally by the mean field $h_{t}$, in analogy to the periodic windows created by an external drive $g$ acting on a single map equation (9). Different initial conditions may lead to different out-of-phase orbits with diverse partitions that appear as clusters in the globally coupled system. A synchronization state in system equations (7) and (9) can be associated to the fixed point interval of the bifurcation diagram of Figure 8(a), while a desynchronization state in the globally coupled system is a manifestation of a chaotic regime as seen in Figure 8(a). Nontrivial forms of the driving function can give rise to multistable behavior besides periodic windows. For example, we have verified that a drive such as $g\left(y_{t}\right)=0.48 f\left(y_{t}\right)+0.4$ in equation (16) induces a region of bistability between chaotic attractors that expresses as chimera states in the associated globally coupled system equations (7) and (9).

These results suggest that the emergence of cluster and chimera states in a globally coupled system of robustchaos maps can be inferred from the occurrence of periodic windows in the response dynamics of a single map subject to an appropriate drive, as a function of parameters. Figure $8(\mathrm{~b})$ shows the corresponding bifurcation diagram of $x_{t+1}=(1-\varepsilon) f\left(x_{t}\right)$ versus $\epsilon$ for the map $f$ given by equation (11) which also has robust chaos. Again, we see the emergence of periodic windows as the coupling parameter is varied. A globally coupled system of these maps also shows clusters and chimera states, as illustrated in Figure 5. Figure 8(c) presents the bifurcation diagram of $x_{t+1}=(1-\varepsilon) f\left(x_{t}\right)$ versus $\varepsilon$ for the logarithmic map $f=a+\ln |x|$, which possesses robust chaos on the parameter interval $a \in[-1,1]$ and its dynamics is unbounded [2]. In contrast to Figures 8(a)and 8(b), no periodic windows appear on the dynamics of the driven map equation (16) which remains unbounded; only chaotic orbits and a fixed point attractor appear. As a consequence, clusters and chimera states should not be expected in a globally coupled system of logarithmic 


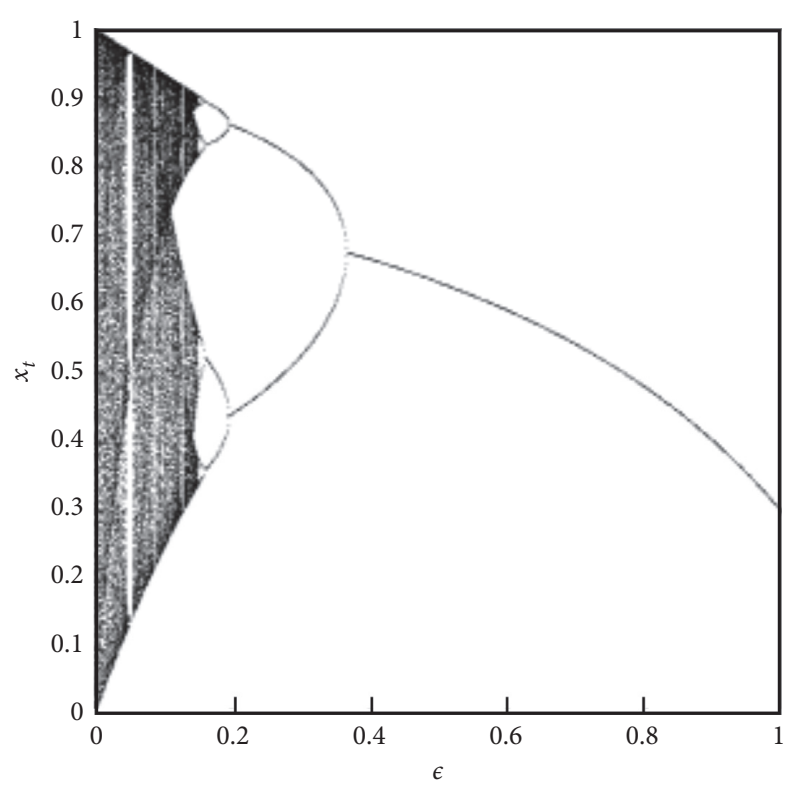

(a)

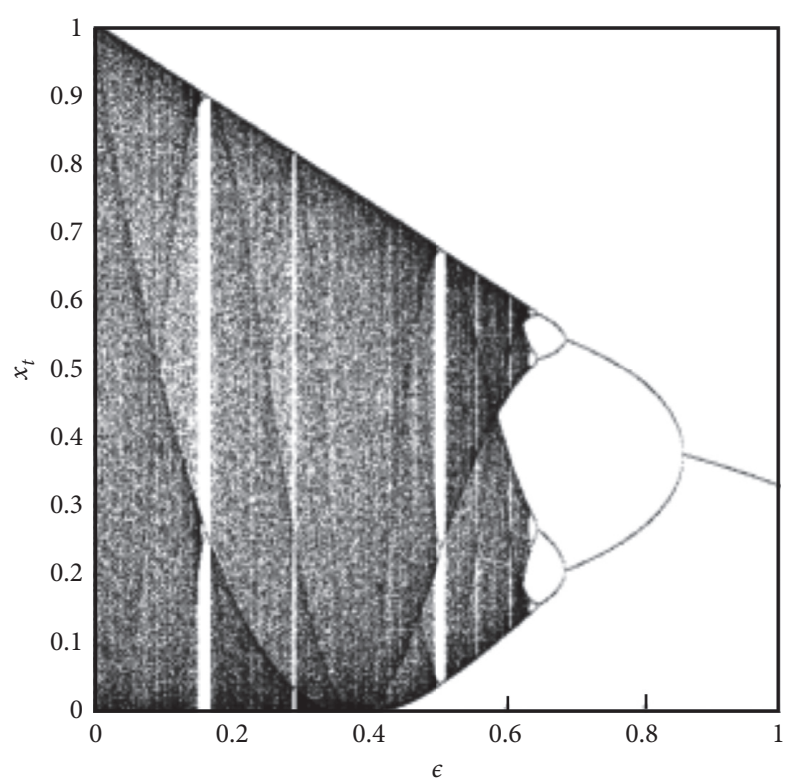

(b)

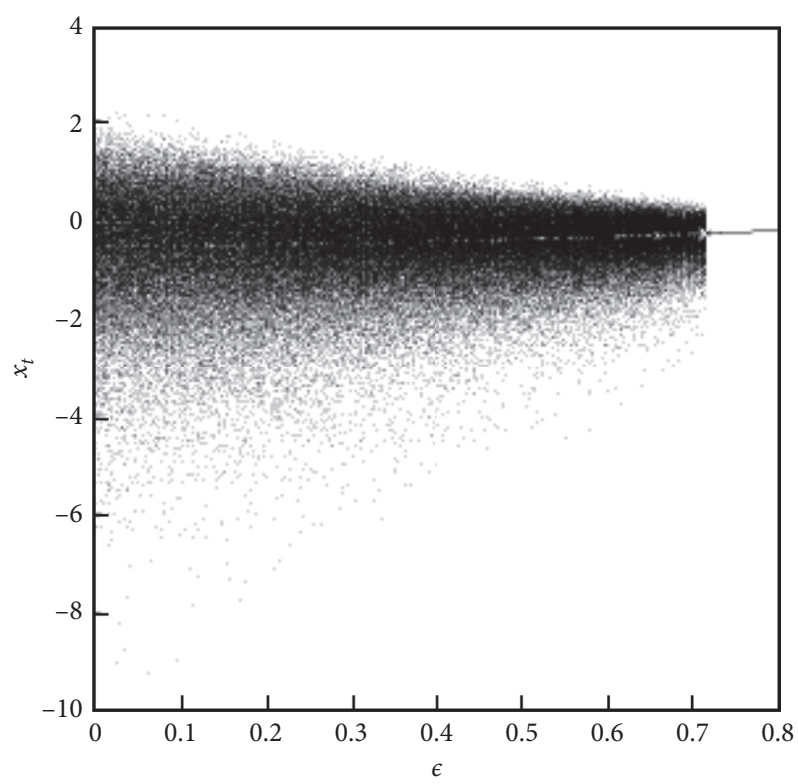

(c)

FIGURE 8: Bifurcation diagrams of the driven map $x_{t+1}=(1-\varepsilon) f\left(x_{t}\right)$ in equation (16) as a function of $\epsilon$ for different robust-chaos maps $f$. (a) $f(x)=\left(1-b^{(1-x) x} / 1-b^{1 / 4}\right)$ with $b=0.5$. (b) $f(x)=\sin ^{2}(r \arcsin (\sqrt{x}))$ with $r=3$. (c) $f(x)=\ln |x|$.

maps. In fact, only synchronization and nontrivial collective behavior have been observed in such a system [57].

\section{Conclusions}

Networks of globally coupled identical oscillators are among the simplest symmetric spatiotemporal systems that can display clustering and chimera behavior. Previous works have conjectured that these phenomena cannot occur when the local oscillators possess robust-chaos attractors [9-13]. We have shown that the presence of global interactions can indeed allow for emergence of both cluster and chimera states in systems of coupled robust-chaos maps. Chimeras appear as partially ordered states between synchronization or clustering and incoherent behavior. We have found that chimera states are associated to the formation of clusters in these systems, a feature that has been observed in other globally coupled systems [31].

The existence of intrinsic periodic windows in the dynamics of local oscillators, such as in logistic maps, is not essential for the emergence of clusters with periodic behavior in a globally coupled system of those oscillators. Windows of periodicity and multistability can be induced in the dynamical response of a robust-chaos map subject to an appropriate external forcing. 
Because of the analogy between a single driven map and the local dynamics of a globally coupled map system, the global interaction field $h_{t}$ can also induce periodic windows and multistability on local robust-chaos maps. Those are the essential ingredients for the occurrence of cluster and chimera states in globally coupled systems. Since clustering is a prerequisite for chimeras [31], a single driven robust-chaos map that develops periodic windows on some range of parameters allows us to infer that a globally coupled system of such maps shall also exhibit cluster and chimera states on some range of parameters. Conversely, a robust-chaos map, such as the logarithmic or another singular map, which does not give rise to periodic windows when subject to a drive, implies that a system of globally coupled logarithmic or singular maps does not show clusters nor chimera states.

Further extensions of this work include the investigation of chimera states in networks of globally coupled continuous-time dynamical systems possessing robust chaos or hyperbolic chaotic attractors, the study of interacting populations of robust-chaos elements, and the role of the range of interaction in a network of dynamical robust-chaos units.

\section{Data Availability}

The programming codes used in this article are available upon request.

\section{Conflicts of Interest}

The authors declare that there are no conflicts of interest regarding the publication of this article.

\section{Acknowledgments}

This study was supported by Corporación Ecuatoriana para el Desarrollo de la Investigación y Academia (CEDIA) through project CEPRA-XIII-2019 "Sistemas Complejos."

\section{References}

[1] S. Banerjee, J. A. Yorke, and C. Grebogi, "Robust chaos," Physical Review Letters, vol. 80, no. 14, p. 3049, 1998.

[2] T. Kawabe and Y. Kondo, Progress of Theoretical Physics, vol. 85, p. 759, 1991.

[3] A. Priel and I. Kanter, "Robust chaos generation by a perceptron," Europhysics Letters (EPL), vol. 51, no. 2, p. 230, 2000.

[4] A. Potapov and M. K. Ali, "Robust chaos in neural networks," Physics Letters A, vol. 277, no. 6, p. 310, 2000.

[5] Z. Elhadj and J. C. Sprott, "On the robustness of chaos in dynamical systems: theories and applications," Frontiers of Physics in China, vol. 3, no. 2, p. 195, 2008.

[6] J. A. C. Gallas, "The structure of infinite periodic and chaotic hub cascades in phase diagrams of simple autonomous flows," International Journal of Bifurcation and Chaos, vol. 20, p. 197, 2010.

[7] P. García, A. Parravano, M. G. Cosenza, J. Jiménez, and A. Marcano, "Coupled map networks as communication schemes," Physical Review E, vol. 65, no. 4, Article ID 45201, 2002.
[8] L. Cisneros, J. Jiménez, M. G. Cosenza, and A. Parravano, "Information transfer and nontrivial collective behavior in chaotic coupled map networks," Physical Review E, vol. 65, no. 4, Article ID 45204, 2002.

[9] M. G. Cosenza and A. Parravano, "Dynamics of coupling functions in globally coupled maps: Size, periodicity, and stability of clusters," Physical Review E.vol. 64, Article ID 36224, 2001.

[10] S. C. Manrubia, A. S. Mikhailov, and D. H. Zanette, "Emergence of dynamical order: synchronization phenomena in complex systems," World Scientific Lecture Notes in Complex Systems, Vol. Vol. 2, World Scientific, Singapore, 2004.

[11] R. Charrier, C. Bourjot, and F. Charpillet, "Flocking as a synchronization phenomenon with logistic agents," in Proceedings of European Conference on Complex Systems, Dresden, Germany, October 2007.

[12] N. Semenova, A. Zakharova, E. Schöll, and V. Anishchenko, "Does hyperbolicity impede emergence of chimera states in networks of nonlocally coupled chaotic oscillators?" EPL (Europhysics Letters), vol. 112, no. 4, Article ID 40002, 2015.

[13] N. Semenova, A. Zakharova, E. Schöll, and V. Anishchenko, "Impact of hyperbolicity on chimera states in ensembles of nonlocally coupled chaotic oscillators," AIP Conference Proceedings, vol. 1738, Article ID 210014, 2016.

[14] K. Kaneko, "Clustering, coding, switching, hierarchical ordering, and control in a network of chaotic elements," Physica D: Nonlinear Phenomena, vol. 41, no. 2, p. 137, 1990.

[15] K. Kaneko, "From globally coupled maps to complex-systems biology," Chaos: An Interdisciplinary Journal of Nonlinear Science, vol. 25, Article ID 97608, 2015.

[16] D. H. Zanette and A. S. Mikhailov, "Condensation in globally coupled populations of chaotic dynamical systems," Physical Review E, vol. 57, no. 1, p. 276, 1998.

[17] D. H. Zanette and A. S. Mikhailov, "Mutual synchronization in ensembles of globally coupled neural networks," Physical Review E, vol. 58, no. 1, p. 872, 1998.

[18] C. Furusawa and K. Kaneko, "Origin of complexity in multicellular organisms," Physical Review Letters, vol. 84, no. 26, p. 6130, 2000.

[19] W. Wang, I. Z. Kiss, and J. L. Hudson, "Experiments on arrays of globally coupled chaotic electrochemical oscillators: synchronization and clustering," Chaos: An Interdisciplinary Journal of Nonlinear Science, vol. 10, no. 1, p. 248, 2000.

[20] K. Miyakawa and K. Yamada, "Synchronization and clustering in globally coupled salt-water oscillators," Physica D: Nonlinear Phenomena, vol. 151, no. 2-4, p. 217, 2001.

[21] E. Schöll, A. Zakharova, and R. G. Andrzejak, Chimera States in Complex Networks, Frontiers Media SA. Ebook., Lausanne, Switzerland, 2020.

[22] M. J. Panaggio and D. M. Abrams, "Chimera states: coexistence of coherence and incoherence in networks of coupled oscillators," Nonlinearity, vol. 28, no. 3, pp. R67-R87, 2015.

[23] Y. Kuramoto and D. Battogtokh, "Coexistence of coherence and incoherence in nonlocally coupled phase oscillators," Nonlinear Phenomena in Complex Systems, vol. 5, p. 380, 2002.

[24] D. M. Abrams and S. H. Strogatz, "Chimera states for coupled oscillators," Physical Review Letters, vol. 93, Article ID 174102, 2004.

[25] C. R. Laing, "Chimeras in networks with purely local coupling," Physical Review Letters, vol. 92, no. R, Article ID 50904, 2015. 
[26] M. G. Clerc, S. Coulibaly, M. A. Ferré, M. A. García-Ñustes, and R. G. Rojas, "Chimera-type states induced by local coupling," Physical Review Letters, vol. 93, Article ID 52204, 2016.

[27] B. K. Bera and D. Ghosh, "Chimera states in purely local delay-coupled oscillators," Physical Review Letters, vol. 93, Article ID 52223, 2016.

[28] J. Hizanidis, N. Lazarides, and G. P. Tsironis, "Robust chimera states in SQUID metamaterials with local interactions," Physical Review Letters, vol. 94, Article ID 32219, 2016.

[29] G. C. Sethia and A. Sen, "The existence criteria revisited," Physical Review Letters, vol. 112, Article ID 144101, 2014.

[30] A. Yeldesbay, A. Pikovsky, and M. Rosenblum, "Chimeralike states in an ensemble of globally coupled oscillators," Physical Review Letters, vol. 112, Article ID 144103, 2014.

[31] L. Schmidt and K. Krischer, "Publisher's note: longitudinal target-spin asymmetries for deeply virtual compton scattering," Physical Review Letters, vol. 114, Article ID 34101, 2015.

[32] A. Mishra, S. Saha, C. Hens et al., "Chimeralike states in a network of oscillators under attractive and repulsive global coupling," Physical Review E, vol. 92, Article ID 62920, 2015.

[33] A. V. Cano and M. G. Cosenza, "Chimeras and clusters in networks of hyperbolic chaotic oscillators," Physical Review E, vol. 95, no. 3, Article ID 30202, 2017.

[34] A. V. Cano and M. G. Cosenza, "Asymmetric cluster and chimera dynamics in globally coupled systems," Chaos: An Interdisciplinary Journal of Nonlinear Science, vol. 28, no. 11, Article ID 113119, 2018.

[35] R. G. Andrzejak, G. Ruzzene, E. Schöll, and I. Omelchenko, "Two populations of coupled quadratic maps exhibit a plentitude of symmetric and symmetry broken dynamics," Chaos: An Interdisciplinary Journal of Nonlinear Science, vol. 30, Article ID 33125, 2020.

[36] I. Omelchenko, B. Riemenschneider, P. Hövel, Y. Maistrenko, and E. Schöll, "Transition from spatial coherence to incoherence in coupled chaotic systems," Physical Review E, vol. 85, Article ID 26212, 2012.

[37] J. Singha and N. Gupte, "Spatial splay states and splay chimera states in coupled map lattices," Physical Review E, vol. 94, Article ID 52204, 2016.

[38] E. V. Rybalova, G. I. Strelkova, and V. S. Anishchenko, "Mechanism of realizing a solitary state chimera in a ring of nonlocally coupled chaotic maps," Chaos, Solitons \& Fractals, vol. 115, p. 300, 2018.

[39] S. Ulonska, I. Omelchenko, A. Zakharova, and E. Schöll, "Chimera states in networks of Van der Pol oscillators with hierarchical connectivities," Chaos: An Interdisciplinary Journal of Nonlinear Science, vol. 26, Article ID 94825, 2016.

[40] J. Hizanidis, V. G. Kanas, A. Bezerianos, and T. Bountis, "Chimera states in networks of nonlocally coupled hindmarsh-rose neuron models," International Journal of Bifurcation and Chaos, vol. 24, no. 3, Article ID 1450030, 2014.

[41] V. M. Bastidas, I. Omelchenko, A. Zakharova, E. Schöll, and T. Brandes, "Quantum signatures of chimera states," Physical Review E, vol. 92, Article ID 62924, 2015.

[42] T. Banerjee, P. S. Dutta, A. Zakharova, and E. Schöll, "Chimera patterns induced by distance-dependent power-law coupling in ecological networks," Physical Review E, vol. 94, Article ID 032206, 2016.

[43] V. Semenov, A. Feoktistov, T. Vadivasova, E. Schöll, and A. Zakharova, "Time-delayed feedback control of coherence resonance near subcritical Hopf bifurcation: Theory versus experiment," Chaos: An Interdisciplinary Journal of Nonlinear Science, vol. 25, Article ID 33111, 2015.
[44] N. C. Rattenborg, C. J. Amlaner, and S. L. Lima, "Behavioral, neurophysiological and evolutionary perspectives on unihemispheric sleep," Neuroscience \& Biobehavioral Reviews, vol. 24 , no. 8 , p. $817,2000$.

[45] A. Rothkegel and K. Lehnertz, "Irregular macroscopic dynamics due to chimera states in small-world networks of pulse-coupled oscillators," New Journal of Physics, vol. 16, Article ID 55006, 2014.

[46] J. C. González-Avella, M. G. Cosenza, and M. San Miguel, "Localized coherence in two interacting populations of social agents," Physica A: Statistical Mechanics and Its Applications, vol. 399, p. 24, 2014.

[47] A. E. Filatova, A. E. Hramov, A. A. Koronovskii, and S. Boccaletti, "Chaos: An Interdisciplinary Journal of Nonlinear Science," Synchronization in networks of spatially extended systems, vol. 18, Article ID 23133, 2008.

[48] M. R. Tinsley, S. Nkomo, and K. Showalter, "Chimera and phase-cluster states in populations of coupled chemical oscillators," Nature Physics, vol. 8, no. 9, p. 662, 2012.

[49] J. D. Hart, K. Bansal, T. E. Murphy, and R. Roy, "Experimental observation of chimera and cluster states in a minimal globally coupled network," Chaos: An Interdisciplinary Journal of Nonlinear Science, vol. 26, Article ID 94801, 2016.

[50] A. M. Hagerstrom, T. E. Murphy, R. Roy, P. Hövel, I. Omelchenko, and E. Schöll, "Experimental observation of chimeras in coupled-map lattices," Nature Physics, vol. 8, no. 9 , p. $658,2012$.

[51] L. Larger, B. Penkovsky, and Y. Maistrenko, "Virtual Chimera States for Delayed-Feedback Systems," Physical Review Letters, vol. 111, Article ID 54103, 2013.

[52] E. A. Martens, S. Thutupallic, A. Fourrierec, and O. Hallatscheka, "Chimera states in mechanical oscillator networks," Proceedings of the National Academy of Sciences. USA, vol. 110, Article ID 10563, 2013.

[53] K. Blaha, R. J. Burrus, J. L. Orozco-Mora et al., "Symmetry effects on naturally arising chimera states in mechanical oscillator networks," Chaos: An Interdisciplinary Journal of Nonlinear Science, vol. 26, no. 11, Article ID 116307, 2016.

[54] M. Andrecut and M. K. Ali, "Robust chaos in smooth unimodal maps," Physical Review E, vol. 64, no. 2, Article ID 25203, 2001.

[55] J. M. Aguirregabiria, "Robust chaos with prescribed natural invariant measure and Lyapunov exponent," 2009, https:// arxiv.org/abs/0907.3790.

[56] I. Omelchenko, O. E. Omelchenko, P. Hövel, and Schöll, "when nonlocal coupling between oscillators becomes stronger: patched synchrony or multichimera states," Physical Review Letters, vol. 110, Article ID 224101, 2013.

[57] M. G. Cosenza and J. González, "Synchronization and collective behavior in globally coupled logarithmic maps," Progress of Theoretical Physics, vol. 100, no. 1, p. 21, 1998. 